\title{
A Pipe Organ-Inspired Ultrasonic Transducer
}

\author{
Alan J. WALKeR* ${ }^{*} \&$ Anthony J. Mulholland ${ }^{\dagger}$ \\ *School of Science and Sport, \\ University of the West of Scotland, \\ Paisley, \\ PA1 2BE, \\ United Kingdom \\ $\dagger$ Department of Mathematics and Statistics, \\ University of Strathclyde, \\ Glasgow, \\ G1 1XH, \\ United Kingdom
}

\begin{abstract}
This article considers a number of backplate designs for the bandwidth improvement of electrostatic ultrasonic transducers in both transmission and reception modes. Motivated by the design of pipe organs, transducers with backplates which incorporate a number of acoustically resonating conduits are modelled using a transmission line mathematical model which describes the displacement of the electrostatic membrane. The model illustrates that by increasing the number and varying the length of these conduits, the transmission voltage response and the reception force response can be improved over the traditional design by around $50 \%$ and $35 \%$, respectively. ultrasound; transducer; pipe organ; electrostatic
\end{abstract}




\section{Introduction}

Ultrasound is employed in a wide variety of applications including medical imaging, non-destructive evaluation, industrial cleaning and SONAR (see Ladabaum et al. (1998) and Leighton (2007)). Ultrasound is used by many animals such as bats (see Amichai et al. (2015)), dolphins (see Au (1993)) and insects (see Barber \& Kawakara (2013)) using highly advanced, nonlinear generation and detection facilities. In the absence of these facilities, humans rely on electrostatic and piezoelectric transducers for the generation and detection of ultrasonic waves (see Manthey et al. (1992) and Warring \& Gibilisco (1985)).

Electrostatic transducers consist of a thin dielectric membrane stretched across a conducting backplate. The backplate is often grooved in order to trap air beneath the membrane and reduce its rigidity (see Schindel et al. (1995)). Recently, ultrasonic transducers with acoustic amplifying conduits emanating from a machined cavity in the backplate have been designed, modelled and tested (see Campbell et al. (2006), Walker et al. (2008), Walker \& Mulholland (2010) and Walker \& Mulholland (2016)). These investigations have shown an improvement on two key factors, namely the gain of the transmission voltage response (TVR) and the reception force response (RFR). However, many applications require not only a high TVR/RFR gain, but a large bandwidth, so that the device can operate over a large frequency range. This would enable these devices to transmit and receive signals with a rich frequency content, such as chirps, as used in the animal world (see Maurello et al. (2000)). It would also improve the axial resolution of these devices as narrower impulses could be generated in the time domain. With this in mind, it is imperative that backplates can be designed which maximise the operational bandwidth of electrostatic transducers.

This article considers a range of designs using an automatic sampling procedure, a self-similar design with a geometric progression of length scales and some designs inspired by the key features of pipe organs. A mathematical model is constructed which outputs the operational bandwidths of the proposed transducers and these are then compared against similar outputs for a standard electrostatic transducer design. We find that all designs which incorporate acoustic amplifying conduits increase the operational bandwidth in the transmission mode and $95 \%$ of the designs increase the operational bandwidth in the reception mode. We also find that while the pipe organ-inspired transducer out-performs the standard transducer, it is in-turn outperformed by a self-similar design with a geometric progression of length scales.

Pipe organ design is considered in Section 2 and then followed by an outline of the mathematical model of the transducer designs in Section 3. The results are presented in Section 4 followed by comments and conclusions in Section 5. 


\section{Pipe Organs}

Pipe organs produce sound by driving pressurised air through a series of pipes via a keyboard. The design of the individual pipes affects the sound produced, with design aspects consisting of length, radii, thickness, construction material, shape, scale (ratio of diameter to length) and presence or absence of a reed (see Bonavia-Hunt (1947), Barnes (1952), Fletcher (1977), Helmholtz (1895), Hopkins (1855), McVicker (1987) and Rucz (2015)). A rank of pipes covers each note on a standard keyboard (normally 61 pipes) and pipe organs generally have a certain amount of ranks (normally at least ten, but often with hundreds). Each rank has a certain type of pipe and by pulling a certain 'stop', the organist can control from which rank of pipes they will hear the key presses. In each rank, the stop refers to the largest pipe in the rank, each successive pipe is then slightly shorter to account for each of the 61 keys on a standard keyboard. For example, the pipe organ situated at the University of Strathclyde's Barony Hall (see Figure 1) has 41 stops, ranging from 16-foot metal Quintadena pipes, through 8-foot metal Dulcian pipes with oak boots, to 4-foot oak Blockflöte pipes with the smallest rank being 2-foot metal Cornet pipes (The University of Strathclyde (2016)). Furthermore, the diameter of each pipe is scaled according to normalmensur. That is, the diameter of each pipe is halved every 17 pipes (depending on type of pipe) where, in this case, 17 is the halving number. In normalmensur, the center $C$ is generally an 8 -foot stop with diameter $155.5 \mathrm{~cm}$ and every other pipe in the rank is calculated with respect to this design. The scaling of the pipes follows the $1: \sqrt[4]{8}$ ratio, or

$$
d_{n}=\frac{d_{1}}{2^{\frac{n-1}{h-1}}},
$$

where $d_{n}$ is the diameter of the $n^{\text {th }}$ pipe and $h$ is the halving number. Each pipe will relate to one specific note on the musical scale and sometimes length-adjusting collars are required in order to get the produced sound to the exact required frequency (see Jones et al. (1941), Nolle (1979) and Fletcher \& Rossing (1991)).

In order to design an array of conduits which are inspired by a pipe organ, we must first choose a pipe organ design. Given that pipe organs vary greatly in size and complexity, we have chosen a small, standard pipe organ (with one console). Such a console would typically have around 610 pipes, with every pipe being different either in length, surface area, shape or construction material. Neglecting reed pipes, closed pipes and tapered pipes, a simplified design could have one rank (61 pipes) each of 16-foot stops, 8-foot stops, 4-foot stops, 2-foot stops and 1-foot stops. With this organ-design in mind, we consider a backplate with five ranks of 61 conduits each. The first rank has a length which has its fundamental frequency at $100 \mathrm{kHz}$, the next rank is halved in length, the next quartered and so on. We use the normalmensur scaling equation (2.1) to compute the radii for each of the 


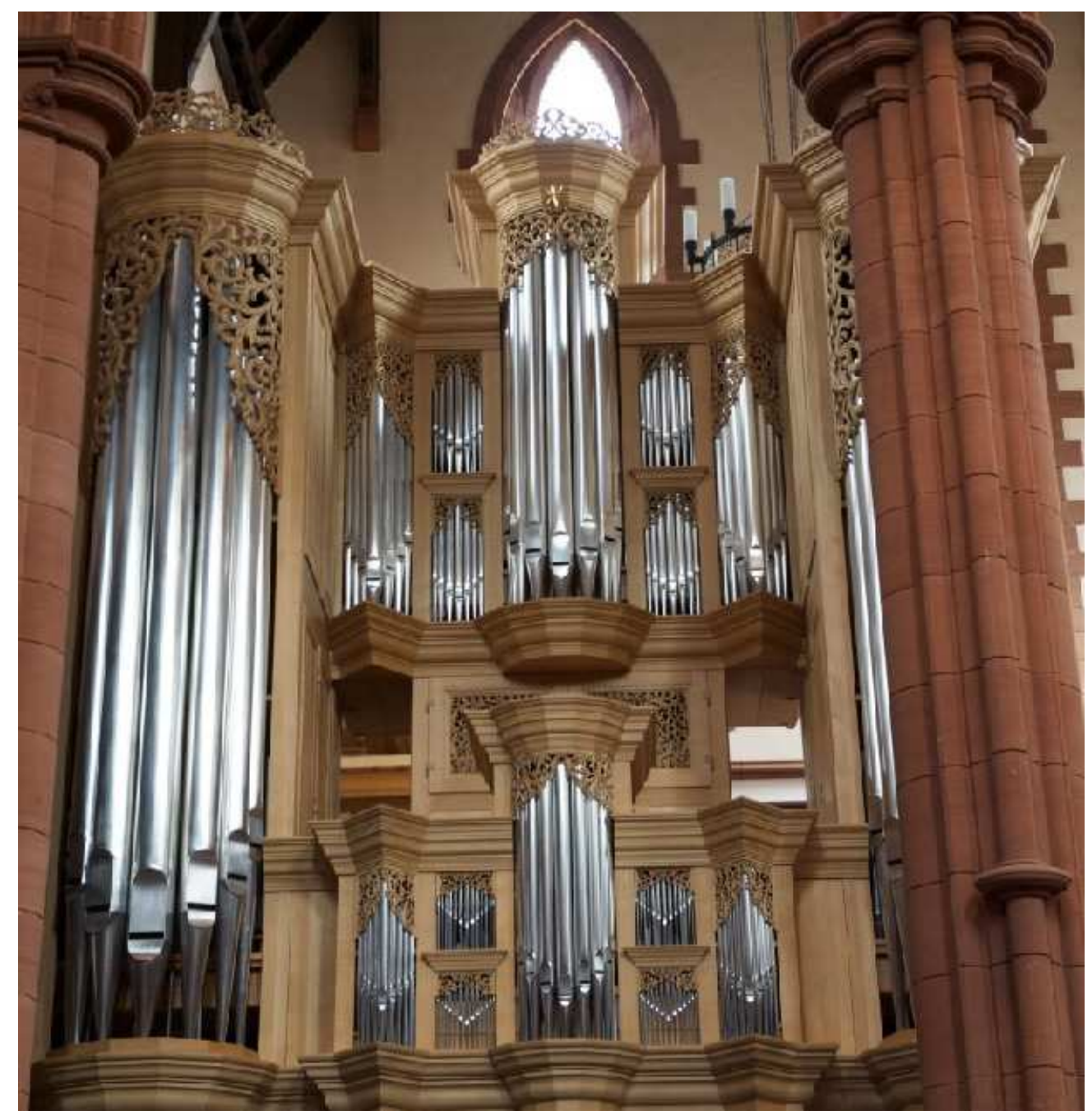

Figure 1: The pipe organ situated at the University of Strathclyde's Barony Hall.

61 conduits in each rank (roughly maximised for maximum surface area). This design, along with a range of other designs will be inserted into the mathematical model for the electrostatic transducer outlined in the next section, and the results shown in Section 4. 


\section{Mathematical Model}

The mathematical model used in this article is a transmission line (1D in space) model introduced by Walker \& Mulholland (2010) and discussed further by Walker $\&$ Mulholland (2016). The model outputs the transmission voltage response (TVR) and the reception force response (RFR) of the ultrasonic transducer, from which the bandwidths are calculated and compared against the standard transducer design. As mentioned, standard electrostatic transducers operate by electrically exciting a stretch mylar membrane over a conducting backplate. The novel design considered in this approach uses acoustic amplifying conduits emanating from an air-filled cavity in the backplate. A simplified exploded sketch of the design is shown in Figure 2.

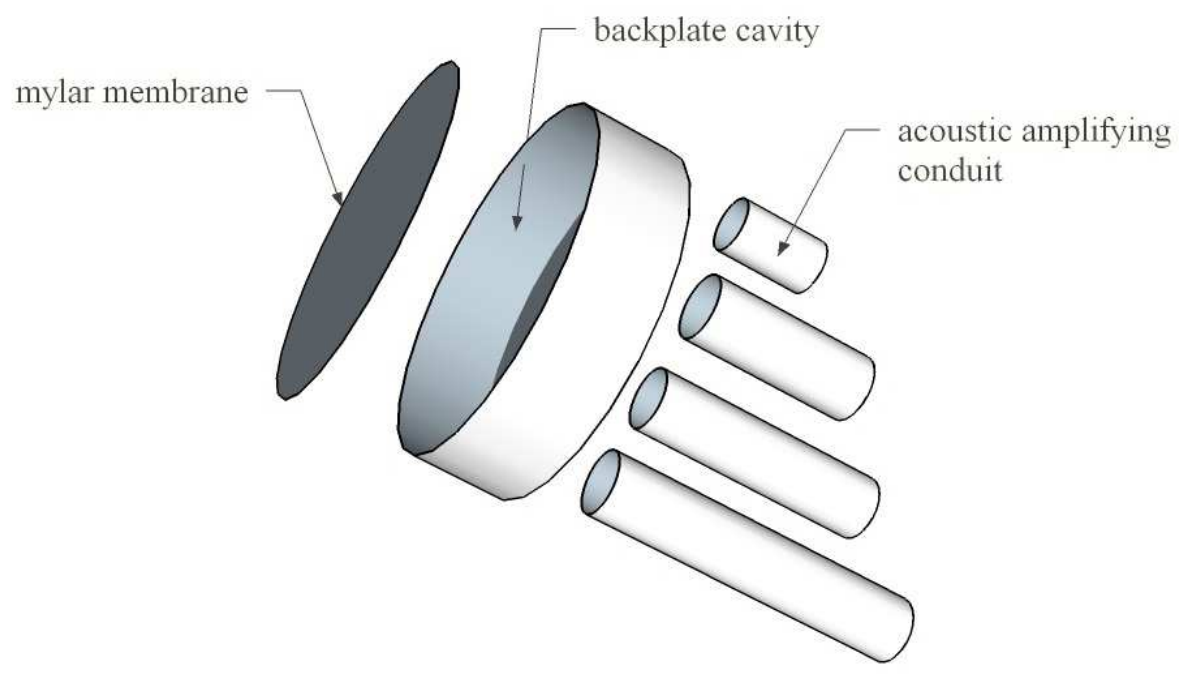

Figure 2: A simplified exploded schematic of the cavity/conduit design. Note that tens or hundreds of acoustic amplifying conduits could emanate from the cavity.

To begin, the radiation impedance for one acoustic amplifying conduit is computed and then used to provide a lumped impedance profile for the entire backplate. This is then inserted into the membrane displacement model which provides the mechanism for computing the bandwidths of the transmission voltage response and the reception force response. 


\subsection{Backplate Impedance Model}

A single acoustic amplifying conduit, consisting of an open cylinder with radius $r_{p}$ and length $l_{p}$, is first considered. Assuming that this conduit represents a straight, open cylindrical pipe of a pipe organ, where the wavelength of the sound is large in comparison to the radius of the conduit (that is, $c / f \gg r_{p}$, validated in Tables 1 and 2), the mechanical radiation impedance, $R_{p}$, at the open end of the conduit is given by (see (Kinsler et al., 2000, p.272-274))

$$
R_{p}=c \rho_{a} \gamma\left(\frac{1}{4}\left(k l_{p}\right)^{2}+0.6 j k l_{p}\right)
$$

where $f$ is the frequency of sound, $c$ is the speed of sound, $\rho_{a}$ is the density of air, $\gamma=\pi r_{p}^{2}$ is the cross-sectional area of the conduit, $k=(1+\alpha j) \omega / c$ is the wavenumber of the sound, with $\alpha$ a nondimensional attenuation coefficient, $\omega$ the angular frequency and $j=\sqrt{-1}$. Consequently, the specific acoustic impedance of the conduit at the conduit/cavity interface, $Z_{s}^{p}$, is given by (see Walker $\&$ Mulholland (2010))

$$
Z_{s}^{p}=\frac{c \rho_{a}\left(R_{p} /\left(c \rho_{a} \gamma\right)+j \tan \left(k l_{p}\right)\right)}{1+j\left(R_{p} /\left(c \rho_{a} \gamma\right)\right) \tan \left(k l_{p}\right)} .
$$

As mentioned, the backplate shall be designed with that of a pipe organ in mind. That is, the backplate shall incorporate an array of conduits. Consequently, each conduit's impedance must be combined to form a lumped acoustic impedance which can then be used to calculate the impedance of the backplate. Defining $Z^{p}[i, j]$ as the acoustic impedance of the conduit in the $i^{t h}$ row and $j^{t h}$ column of the array of conduits, the lumped acoustic impedance of the conduits is given by (see (Kinsler et al., 2000, p.288-291))

$$
Z_{p}=\frac{1}{\sum_{j=1}^{N} \sum_{i=1}^{n_{j}} 1 / Z^{p}[i, j]},
$$

where $n_{j}$ is the number of conduits in row $j$ and $N$ is the number of rows in the conduit array. Consequently, the specific acoustic impedance in the cavity, $Z_{s}^{c}$, can be found via

$$
Z_{s}^{c}=\frac{c \rho_{a}\left(Z_{p} /\left(c \rho_{a} S_{c}\right)+j \tan \left(k l_{c}\right)\right)}{1+j\left(Z_{p} /\left(c \rho_{a} S_{c}\right)\right) \tan \left(k l_{c}\right)},
$$

where $S_{c}$ is the surface area of the cavity and $l_{c}$ is the length of the cavity. This impedance can then be used in the transmission line model for the displacement of the mylar membrane. 


\subsection{Transmission Line Model of Membrane Displacement}

As shown by Walker \& Mulholland (2016), the displacement of the membrane can be modelled via a pipe-driver system, a membrane model or a plate model. As each of these model produces similar results, we shall use the pipe-driver system detailed by Kinsler et al. (2000). Here, the membrane is assumed to act like a damped harmonic oscillator, which is excited by an external force $f(t)$. Following the analysis by Walker et al. (2008), Walker \& Mulholland (2010) and Walker \& Mulholland (2016), the dynamic equation for the membrane displacement $\xi$ is given by

$$
d_{m} \rho_{s} \ddot{\xi}+\left(\frac{R_{v}}{S_{m}}+Z_{s}^{l}+\frac{S_{c}}{S_{m}} Z_{s}^{c}\right) \dot{\xi}+\left(\frac{S_{c} Z_{m}^{c}}{S_{m} V_{c}}-\frac{\varepsilon_{0} V_{d c}^{2}}{d_{e}^{3}}\right) \xi=f(t),
$$

where $d_{m}$ is the thickness of the membrane, $\rho_{s}$ is the density of the membrane, $R_{v}$ is a damping constant, $S_{m}$ is the surface area of the membrane, $Z_{s}^{l}$ is the specific acoustic impedance of the fluid load, $Z_{m}^{c}$ is the mechanical impedance of the cavity, $V_{c}$ is the volume of the cavity, $\varepsilon_{0}$ is the permittivity of free space, $V_{d c}$ is the direct current voltage, $d_{e}$ is the distance between the electrodes and an overhead dot represents the time derivative. The external force, which combines the voltage driving force applied to the membrane and any incoming pressure wave $P(t)$, is given by

$$
f(t)=\frac{\varepsilon_{0} V_{d c} V_{a c}(t)}{d_{e}^{2}}+P(t),
$$

where $V_{a c}(t)$ is the a.c. voltage. Clearly, $V_{a c}(t)=0$ in reception mode and $P(t)=0$ in transmission mode. Taking the Fourier transform (see Wright (2005)) of the differential equation (3.5) gives

$$
\Xi(\omega)=\frac{1}{j \omega Z_{s}^{m}(\omega)} \bar{f}(\omega),
$$

where $\Xi(\omega)$ is the displacement of the membrane in the frequency domain,

$\bar{f}(\omega)=\varepsilon_{0} V_{d c} \bar{V}_{a c}(\omega) / d_{e}^{2}+\bar{P}(\omega)$, with $\bar{V}_{a c}(\omega)$ the alternating current voltage in the frequency domain, $\bar{P}(\omega)$ the incoming pressure wave in the frequency domain and

$$
Z_{s}^{m}(\omega)=j d_{m} \rho_{s}+\left(\frac{R_{v}}{S_{m}}+Z_{s}^{l}+\frac{S_{c}}{S_{m}} Z_{s}^{c}\right)-\frac{j}{\omega}\left(\frac{S_{c} Z_{m}^{c}}{S_{m} V_{c}}-\frac{\varepsilon_{0} V_{d c}^{2}}{d_{e}^{3}}\right),
$$

is the specific acoustic impedance of the combined membrane/load system. Consequently, the velocity of the membrane in the frequency domain is

$$
\dot{\Xi}(\omega)=\frac{1}{Z_{s}^{m}(\omega)} \bar{f}(\omega) .
$$


The velocity of the mylar membrane's deflections can then be used to compute the electrical impedance and hence the transmission and reception sensitivities of the device, as seen in the following section.

\subsection{Electrical Impedance, Transmission and Reception Sensi- tivities of the Device}

A transducer converting electrical and mechanical energy forms a two-port network that relates electrical quantities at one port to mechanical quantities at the other (see Kinsler et al. (2000)). The canonical equations which describe this are given by

$$
\bar{V}_{a c}=Z_{E B} I+T \dot{\Xi}, \quad F=T I+Z_{m o} \dot{\Xi},
$$

where $I$ is the current at the electrical inputs, $F$ is the force on/from the radiating surface, $Z_{E B}$ is the blocked electrical impedance $(\dot{\Xi}=0), Z_{m o}$ is the open-circuit mechanical impedance $(I=0)$ and $T$ is the transduction coefficient (mechanical $\leftrightarrow$ electrical). In the short circuit case $\bar{V}_{a c}=0$ it can be shown

$$
\frac{F}{\dot{\Xi}}=\left(Z_{m o}-\frac{T^{2}}{Z_{E B}}\right)=Z_{m},
$$

where $Z_{m}$ is the mechanical impedance of the transducer. Hence equations (3.10) can be rewritten as

$$
\bar{V}_{a c}=Z_{E B} I+\beta Z_{E B} \dot{\Xi}, \quad F=\beta Z_{E B} I+Z_{m} \dot{\Xi},
$$

where the transformation factor $\beta$ is given by $\beta=T / Z_{E B}$. When the source voltage is of the form $V_{a c}(t)=V_{a c} e^{i \omega t}$, then by Caronti et al. (2002), Kinsler et al. (2000) and Walker \& Mulholland (2010)

$$
V_{a c}=\left(\frac{1}{G+i \omega C_{0}}\right) I+\frac{V_{d c}}{i \omega x_{d c}} \dot{\Xi}
$$

where $G$ is the static conductance caused by electrical losses in the device, $C_{0}$ is the value of the capacitance $C$ at $\dot{\Psi}=0$ and $x_{d c}$ is the deflection of the membrane caused by the d.c. voltage. Hence, comparing with equations (3.10) the transformation factor $\beta$ can be found (see Walker \& Mulholland (2010)) and, consequently, the blocked electrical impedance is given by

$$
Z_{E B}=\frac{1}{G+i \omega C_{0}},
$$


where $C_{0}=\varepsilon_{0} S_{m} /\left(d_{m} / \varepsilon_{r}+L+x_{d c}\right)$, with $\varepsilon_{r}$ the relative permitivitty of the membrane and $L$ the distance between electrode and membrane.

In transmission mode, a voltage $\bar{V}_{a c}$ is applied that results in a membrane velocity $\dot{\Xi}$ and hence a force $F$ being produced, where $F=-Z_{m} \dot{\Xi}$. The transmission sensitivity, or transmission voltage response (TVR), is defined as the ratio of the transmitted pressure to the driving voltage (see Caronti et al. (2002)) and after some some algebraic manipulation, it can be shown that (see Walker \& Mulholland (2010))

$$
\operatorname{TVR}=\frac{-\omega V_{d c} C_{0}+i G V_{d c}}{\omega x_{d c}\left(1+S_{m} Z_{s}^{m} / Z^{l}\right)}
$$

This can now be evaluated using the specific acoustic impedance of the combined membrane/load system, given in (3.8), and the acoustic impedance of the load, given by Kinsler et al. (2000) as

$$
Z^{l}=\frac{\rho_{a} c}{S_{m}}\left(1-\frac{J_{1}\left(2 k \sqrt{S_{m} / \pi}\right)}{k \sqrt{S_{m} / \pi}}+j \frac{H_{1}\left(2 k \sqrt{S_{m} / \pi}\right)}{k \sqrt{S_{m} / \pi}}\right)
$$

where $J_{n}$ is the Bessel function of the first kind of order $n$ and $H_{n}$ is the Struve function of order $n$.

The reception sensitivity is defined as the ratio of the open-circuit $(I=0)$ output voltage to the force on the membrane (see Caronti et al. (2002)). That is, the reception force response (RFR) is given by RFR $=\bar{V}_{a c} /\left(P_{o} S_{m}\right)$, where $P_{o}$ is the pressure produced at the membrane load. Setting $I=0$ (for the open-circuit) in equations (3.10) gives

$$
\bar{V}_{a c}=T \dot{\Xi}, \quad F=Z_{m o} \dot{\Xi} .
$$

Since $F=P_{o} S_{m}$ then $\dot{\Xi}=P_{o} S_{m} / Z_{m o}$ and so it can be shown that (see Walker \& Mulholland (2010))

$$
\mathrm{RFR}=\frac{\Xi Z_{E B}}{S_{m} Z_{s}^{m}+T^{2} / Z_{E B}} .
$$

This can be evaluated via equation (3.14) and the specific acoustic impedance of the combined membrane/load system, given in (3.8), which is highly dependent on the design of the transducer's backplate.

\section{Comparison of Transducer Designs}

The success of the pipe organ-inspired transducer described in Section 2 is measured via comparisons against the standard cavity-only transducer, the best performing design from a random sampling of pipe distributions and from some other 
specific designs as described below. In order to successfully compare the devices, the material and other parameter values must be considered. Initial endeavours in manufacturing a variety of backplates have taken place by Hamid (2013) and Campbell et al. (2006) and form the basis for the backplate design parameter values given in Table 1. The (constant) radius of the conduit(s) is chosen so that up to 100 conduits could fit in the backplate (see The Engineering Toolbox (2016)). Additional design and material parameter values are given in Table 2.

\begin{tabular}{lccc}
\hline Design parameter & Symbol & Magnitude & Dimensions \\
\hline Thickness of membrane & $d_{m}$ & 8 & $\mu \mathrm{m}$ \\
Length of cavity & $l_{c}$ & 35 & $\mu \mathrm{m}$ \\
Radius of conduit(s) & $r_{p}$ & 26 & $\mu \mathrm{m}$ \\
Surface area of membrane & $S_{m}$ & $\pi \times 300^{2}$ & $\mu \mathrm{m}^{2}$ \\
Surface area of cavity & $S_{c}$ & $\pi \times 300^{2}$ & $\mu \mathrm{m}^{2}$ \\
\hline
\end{tabular}

Table 1: Standard design values of the backplate.

\begin{tabular}{lccc}
\hline Design Parameter & Symbol & Magnitude & Dimensions \\
\hline Speed of Sound in Air & $c$ & 343 & $\mathrm{~m} / \mathrm{s}$ \\
Damping Coefficient & $R_{v}$ & 100 & $\mathrm{~kg} / \mathrm{m} \mathrm{s}$ \\
Applied Voltage & $V_{a c}$ & 200 & $\mathrm{~V}$ \\
d.c. Voltage & $V_{d c}$ & 200 & $\mathrm{~V}$ \\
d.c. Deflection on membrane & $x_{d c}$ & 60 & $\mathrm{~nm}$ \\
Attenuation Coefficient & $\alpha$ & 0.001 & - \\
Permittivity of Free Space & $\varepsilon_{0}$ & $8.85 \times 10^{-12}$ & $\mathrm{~F} / \mathrm{m}$ \\
Membrane Dielectric Constant & $\varepsilon_{r}$ & 5 & - \\
Density of Air in Resonator & $\rho_{a}$ & 1.2 & $\mathrm{~kg} / \mathrm{m}^{3}$ \\
Density of Mylar Membrane & $\rho_{s}$ & 1420 & $\mathrm{~kg} / \mathrm{m}^{3}$ \\
\hline
\end{tabular}

Table 2: Parameter and design values of the transducer.

We note here a justification of the lumped impedance model, described in Section 3, with the backplate design parameter values, given in Table 1. Equation (3.1) refers to the mechanical radiation impedance of each conduit. These conduits have a radius which is $26 \mu \mathrm{m}$ and this is many times smaller than the acoustic wavelength in air at $400 \mathrm{kHz}$, which is of the order of millimeters. The frequency range of interest is from $100 \mathrm{kHz}$ to $400 \mathrm{kHz}$ and so the corresponding wavelength range is from $800 \mu \mathrm{m}$ to $3430 \mu \mathrm{m}$. The wavelength is also much greater than the radius of the backplate which sits at $300 \mu \mathrm{m}$. Hence, the requirement that $c / f \gg r_{p}$, introduced by Kinsler et al. (2000), is achieved. 
In order to compare the different designs, the operational bandwidth of each device's TVR and RFR is considered. For the TVR comparison, we let $A$ be the set of frequencies $f$ for which the TVR sensitivity lies above a certain threshold. That is,

$$
A=\left\{\left[a_{i}, b_{i}\right], i=1, \ldots, N: \operatorname{TVR}(f)>\tau, \forall f \in\left[a_{i}, b_{i}\right]\right\} .
$$

Here, the threshold $\tau$ is defined to be $-3 \mathrm{~dB}$ below the maximum TVR sensitivity of the standard design, which is described fully below. The operational bandwidth of the TVR of each device is then defined as

$$
B W=\sum_{i=1}^{N}\left(b_{i}-a_{i}\right) .
$$

The comparison of the RFR for each device follows a similar approach. Of course, care must be taken here as the set $A$ could consist of a series of disjoint intervals and so the frequency range over which the device is to be operated within could contain some bandgaps. Nevertheless, it is important to illustrate the total range of frequencies for which these new proposed devices outperform the standard devices. Note also that no damping has been included in either model and so, when introduced, this will serve to smooth out the peaks in these plots.

\subsection{Standard Design}

The standard cavity-only design has a closed cavity with length and surface area specified in Table 1 . The bandwidth at the half-power point $(-3 \mathrm{~dB})$ is calculated for each metric and found to be around $255 \mathrm{kHz}$ and $187 \mathrm{kHz}$ for the TVR and RFR, respectively. It is these values which the new designs will be compared against, as illustrated in Equations (4.1) and (4.2). Plots of the TVR and RFR for the standard design can be found in Figures 4 (dotted line) and 6 (dash-dot-dot line).

\subsection{Sampled Designs}

Five thousand different designs were sampled where each design is given a random number of conduits between 10 and 100. Each conduit has the same radius, stipulated in Table 1 but each conduit length was randomly chosen from a linear distribution with $l_{p} \in[0.4 \mathrm{~mm}, 1.4 \mathrm{~mm}]$. The TVR and RFR bandwidths are calculated and plotted against the number of conduits in Figure 3. We find that, in general, the more conduits present, the larger the bandwidth (although this trend does seem to have a limit). The ten devices with the largest bandwidths have a number of conduits from the set $\{79,80,84,90,93,94,95,97,99\}$ in the transmission mode and from $\{46,47,50,51,61,63,65,66\}$ in the reception mode. 
The ten devices with the smallest bandwidths have a number of conduits from the set $\{10,11,12\}$ in transmission mode and from $\{10,11,12,14\}$ in reception mode. In fact, changing the sampling procedure to choose from between 1-100 conduits means that the smallest ten bandwidths come from designs with a single figure number of conduits. Nevertheless, we see from Figure 3 that in transmission mode, every design outperforms the standard cavity-only device by at least $34 \mathrm{kHz}$ (10 pipes) and up to $134 \mathrm{kHz}$ (79 pipes) - an improvement of around 53\%. In reception mode, we find that $95 \%$ of the sampled designs outperform the standard design, by $65 \mathrm{kHz}$ in the best case (61 pipes), which equates to an improvement of around $35 \%$. The worst case (10 pipes) shows a reduction in the bandwidth of $3 \mathrm{kHz}$, which equates to a reduction in bandwidth by around $5 \%$. The TVR and RFR of the devices with the largest and smallest bandwidths are plotted in Figure 4 alongside the cavity-only standard design, for comparison. We see that the inclusion of many pipes with different lengths creates multiple resonances which leads to a broader spectrum.

A frequency plot of the lengths of the conduits in the best and worst transmission designs is presented in Figure 5. We note that there is no definite pattern as to what design produces the largest bandwidth for the TVR or RFR. The only clear metric which significantly changes the bandwidth is the number of pipes. However, it could be postulated that a distribution of pipes where a linear progression of lengths is employed could fit much of the profile for best TVR and RFR seen in Fig. 5.

With this in mind, we now consider a number of specific designs which are made in order to test the hypothesis that the radii of the pipes are of no real significance, and that a linear array of pipe lengths should produce the largest bandwidth due to the coupling effect of resonances at many different frequencies.

\subsection{Specific Designs}

Three further designs are considered: design $A$ involves 100 conduits all of constant length but with radii chosen according to the normalmensur equation (2.1) and design $B$ involves 100 conduits all of constant radii, but with a linear progression of pipe lengths in the range $l_{p} \in[0.4 \mathrm{~mm}, 1.4 \mathrm{~mm}]$. Design $C$, inspired by the pipe organ, was detailed in Section 2: there are five ranks of 61 conduits each, the conduits in each rank have a length that is half of the previous rank, with the first rank designed to resonate at $100 \mathrm{kHz}$. Furthermore, the radius of the conduit in each rank is scaled according to the normalmensur scaling equation(2.1).

Design $A$ acts as expected with a regular resonant structure as seen in Figure 6 (dotted line). Due to the lack of variance in conduit length, no coupling of resonances is evident and hence the bandwidth in transmission mode is around $330 \mathrm{kHz}$. Clearly this is still an improvement (of $75 \mathrm{kHz}$, almost $30 \%$ ) over the 


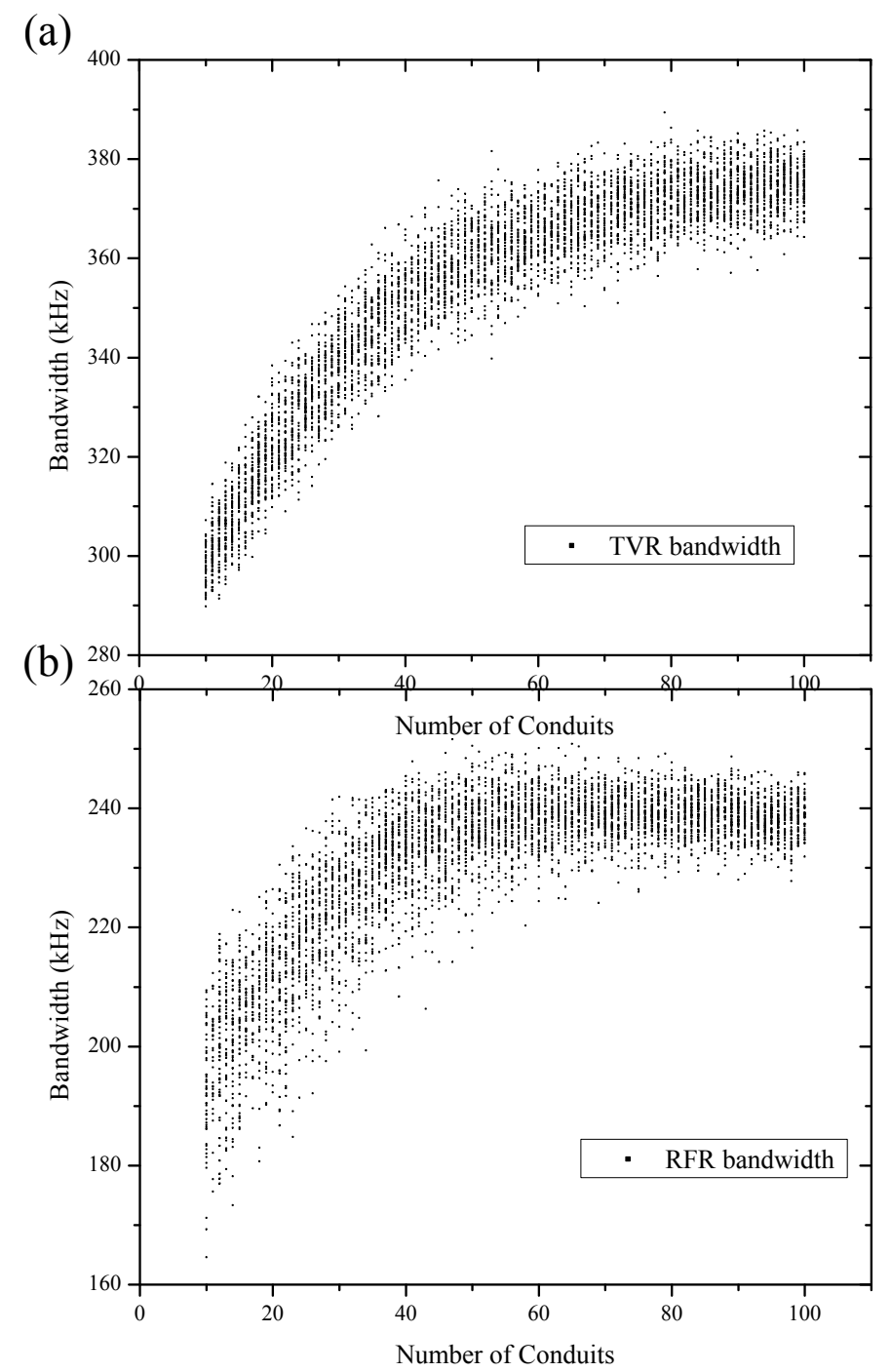

Figure 3: TVR (a) and RFR (b) bandwidths of 5000 samples of device where the number of pipes is randomly sampled between 10 and 100. Each pipe has a length between $0.4 \mathrm{~mm}$ and $1.4 \mathrm{~mm}$ and a radius of $26 \mu \mathrm{m}$. We see that, in general, more pipes produce an improved bandwidth.

standard design with no conduits but illustrates that variety in conduit length is required.

Design $B$ shows a marked improvement over the standard design. We see from Figure 6 (dash-dot line) that the TVR features many resonances around the main operating frequency due to the addition of conduits bearing resonances in this range. The bandwidth of the TVR is found to be around $380 \mathrm{kHz}$, an improvement of almost $50 \%$ over the standard transducer. This suggests that, with 


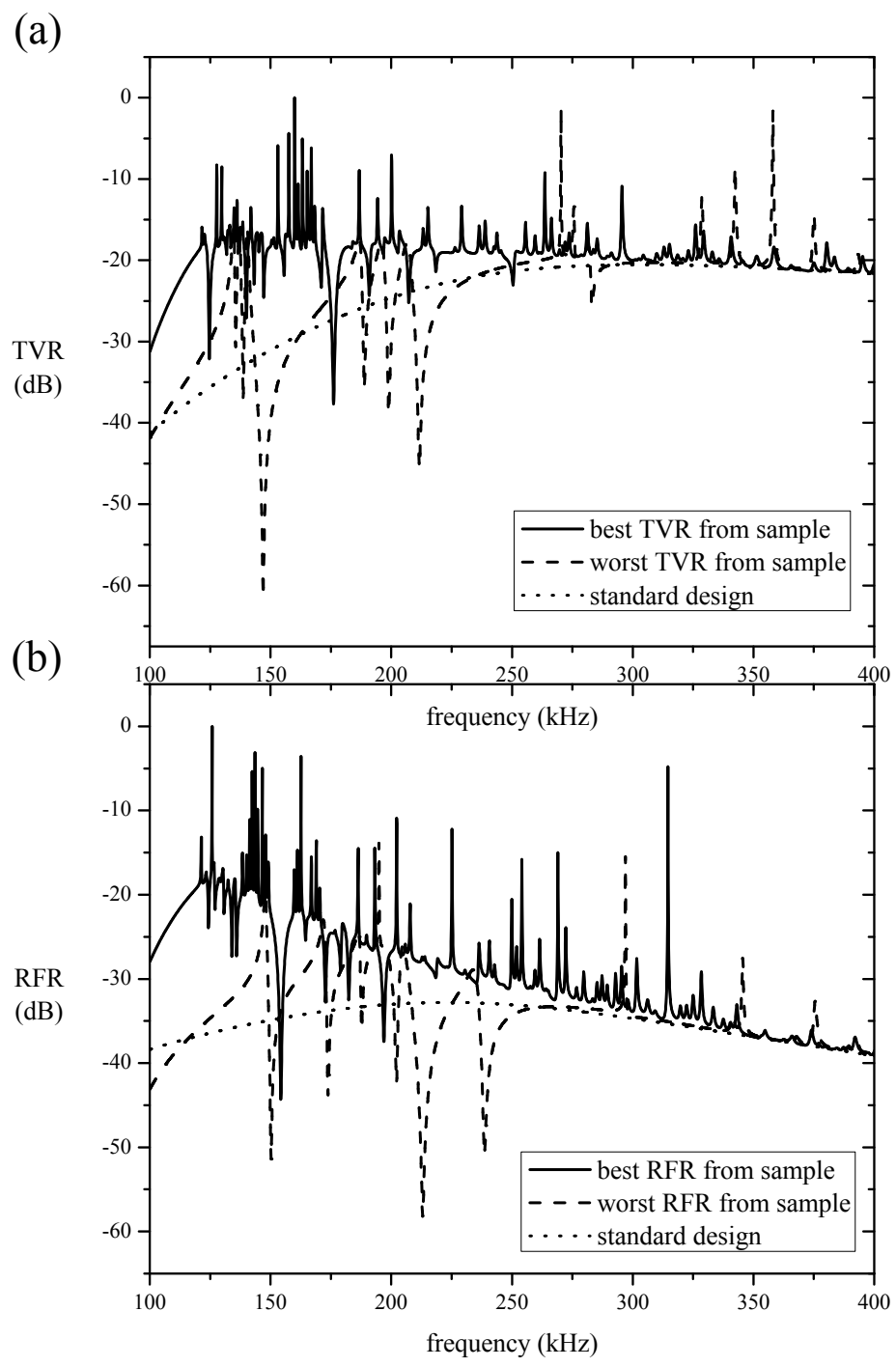

Figure 4: Best (solid) and worst (dashed) TVR (a) and RFR (b) against frequency from 5000 transducer design samples. Also included are outputs from the standard design (dotted).

an appropriate choice of conduit number and dimensions, the backplate can be designed to boost the device's operating bandwidth at a desired frequency range.

For the pipe organ-inspired design $(\operatorname{design} C)$, we see that the increase in the number of pipes has increased the gain of the TVR. However, the lack of variety in conduit lengths results in a lack of coupling of resonances and hence the bandwidth of the TVR is $374 \mathrm{kHz}$. Again, this is a large improvement (of $119 \mathrm{kHz}$, almost $47 \%$ ) over the standard device, but is not quite as impressive as the linear 


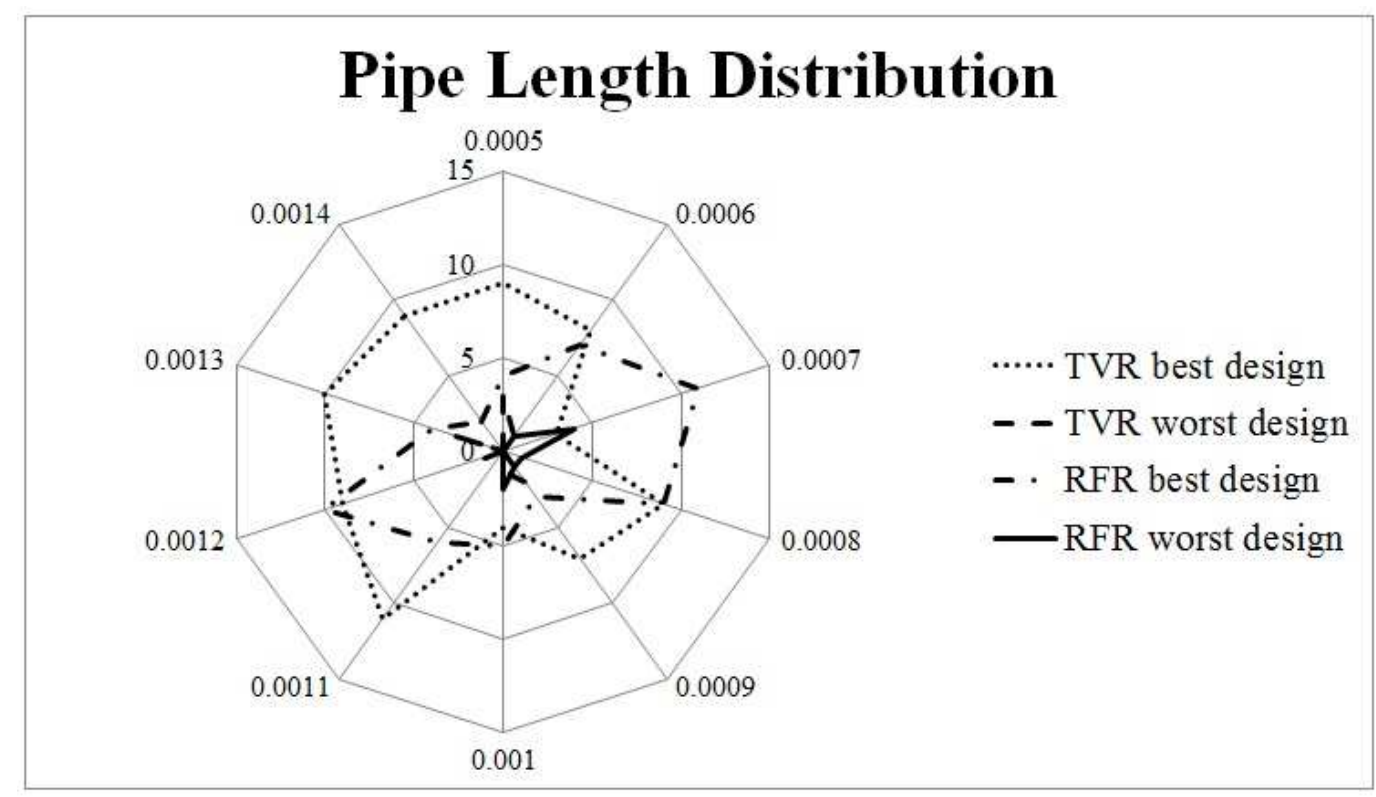

Figure 5: Frequency plots of the best TVR (dotted), best RFR (dash-dot), worst TVR (dash) and worst RFR (solid) devices from a sample of 5000 where the number of pipes is randomly sampled between 10 and 100. Each pipe has a length between $0.4 \mathrm{~mm}$ and $1.4 \mathrm{~mm}$ and a radius of $26 \mu \mathrm{m}$. The best design has 79 pipes in the transmission mode and 61 in the reception mode. The worst has 10 pipes in the transmission mode and 10 in the reception mode. No obvious pattern can be seen in the best case histogram other than more pipes generally equates to a better output.

distribution of pipes, nor the best randomly sampled device.

The bandwidth calculations in reception mode following a similar pattern. De$\operatorname{sign} A$ is hampered by the lack of variety in length scales, with no obvious resonance coming from the variety in pipe radii. The bandwidth in reception mode is $213 \mathrm{kHz}$, an improvement of $26 \mathrm{kHz}$ (almost 14\%). As in transmission mode, design $B$ shows a substantial improvement over the standard design with a reception mode bandwidth of $240 \mathrm{kHz}$ which equates to an improvement of $53 \mathrm{kHz}$ (almost $30 \%$ ). Finally, the pipe organ-inspired design (design $C$ ) also has an improvement over the standard design with a reception mode bandwidth of $222 \mathrm{kHz}$. This equates to an improvement of $35 \mathrm{kHz}$ (almost 19\%). For clarity, the TVR and RFR bandwidths for each device are compared in Table 3. 


\begin{tabular}{lcc}
\hline Design & $\begin{array}{c}\text { TVR bandwidth } \\
(\mathrm{kHz})\end{array}$ & $\begin{array}{c}\text { RFR bandwidth } \\
(\mathrm{kHz})\end{array}$ \\
\hline standard & 255 & 187 \\
A: constant length & 330 & 213 \\
B: linear length distribution & 380 & 240 \\
C: pipe organ & 374 & 222 \\
best from sampling procedure & 390 & 252 \\
\hline
\end{tabular}

Table 3: TVR and RFR bandwidths for standard design, specific designs and best design from the sampling procedure.

\section{Comments and Conclusions}

This article considers a number of backplate designs for the bandwidth improvement of electrostatic ultrasonic transducers. Based on the design of pipe organs, a transducer backplate design is presented and the transducer's transmission voltage response and reception force response are investigated. The output metrics are compared with that of a standard backplate design, some randomly sampled designs and some specific designs which incorporate acoustic amplifying conduits. We find that all designs which incorporate acoustic amplifying conduits emanating from the backplate have a greater transmission mode bandwidth than that of the cavity-only device. This is due to the resonant behaviour of the conduits, which amplify the signal at specific frequencies. By incorporating conduits which have a range of length scales, the individual resonances couple to provide a signal with a wide bandwidth with up to a $50 \%$ improvement on the cavity-only device. It is seen that, in general, the more conduits there are (of varying lengths), the greater the bandwidth of the output signal. Specific designs for specific outputs (be that large gain at a particular frequency, or large bandwidth over a particular frequency range) can be modelled. The reception mode analysis shows a similar improvement over the standard design. Improvements can be found of up to around 30\% and, the designs can be tuned to operate over a specific bandwidth range.

This article makes use of a transmission line (1D in space) mathematical model which describes the displacement of the electrostatic membrane. This is then used to compute the output pressure, transmission voltage response and reception force response. This lumped impedance model is an approximation of the actual air flow in the device, but being computationally fast it allows for the consideration of many thousands of designs. Nevertheless, while the model has shown to accurately portray the pressure output of the device (see Walker \& Mulholland (2010)), experimental prototyping of the proposed design(s) is required and this is now the subject of our ongoing investigations. Such prototypes will 


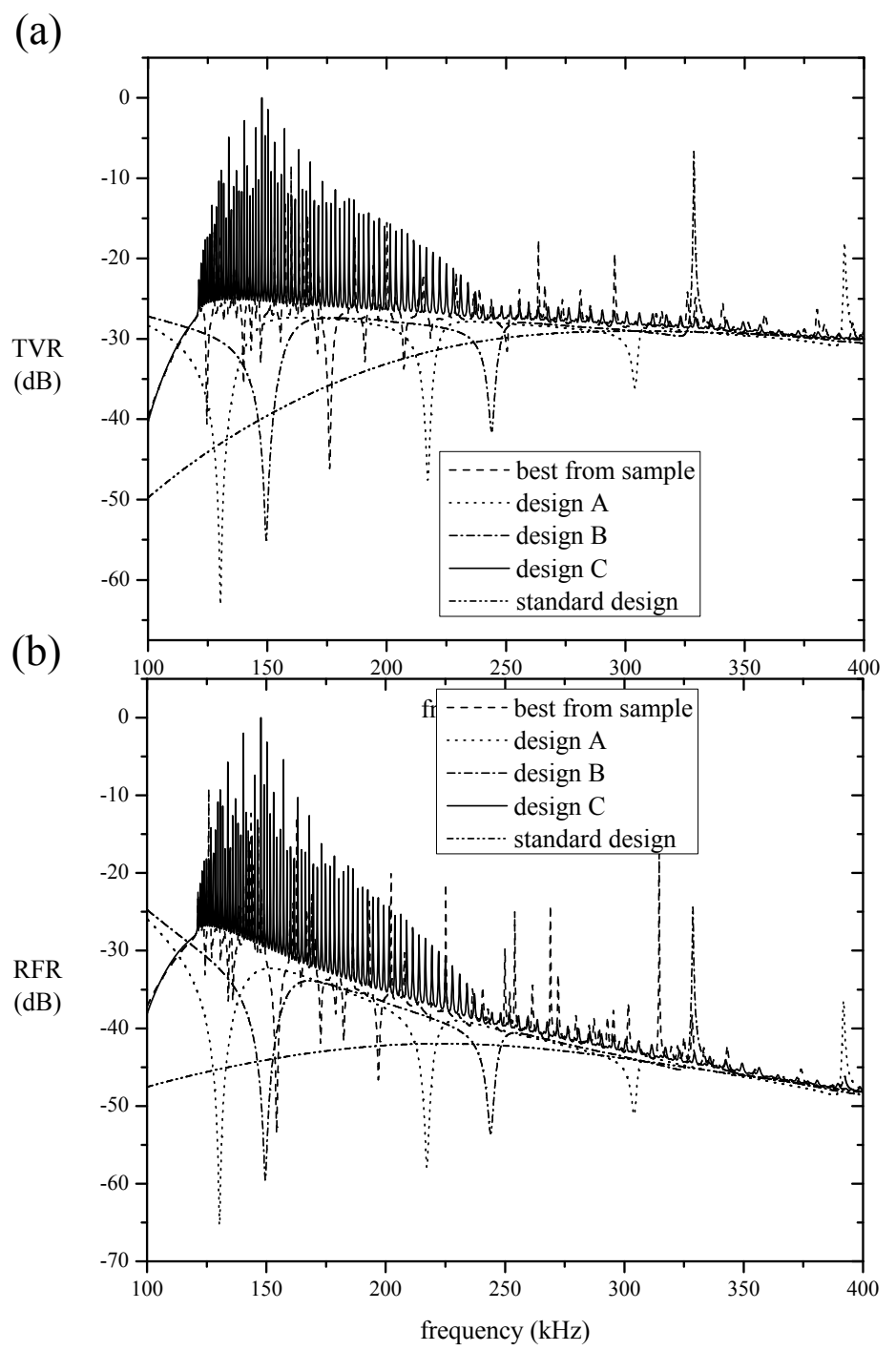

Figure 6: (a) TVR (dB) against frequency $(\mathrm{kHz})$ for four specific designs (with conduits) and the standard design (no conduits), and (b) RFR (dB) against frequency $(\mathrm{kHz})$ for four specific designs (with conduits) and the standard design (no conduits).

make use of rapid prototyping of the backplates and shall allow for some validation of the mathematical model. Furthermore, they will allow for aspects such as the $2 \mathrm{D}$ internal air pressure and beam profile, which are not available in this $1 \mathrm{D}$ model, to be investigated.

This paper could also be used to expand previously published research concerning spherical resonators in backplates (see Walker \& Mulholland (2016)). 
That is, similar sampling procedures could be put in place in order to test for the optimum design of these backplates. Whether these backplates can be built using rapid prototyping, instead of selective dissolution of polymer phases, remains to be seen. However, the advances in rapid prototyping technology allows for a multitude of new backplate designs to be considered.

This paper illustrates how using Monte Carlo sampling over many putative designs can provide a single design which far outperforms the standard device. This could be further improved by optimising the backplate design using a gradient based or stochastic optimisation algorithm and is the subject of ongoing work.

\section{References}

[ONLINE] AVAILABLE AT:

HTTP://WWW.ENGINEERINGTOOLBOX.COM/SMALLER-CIRCLES-IN-

LARGER-CIRCLE-D_1849.HTML

[ACCESSED 25 OCT. 2016].

[ONLINE] AVAilable at:

HTTP://WWW.STRATH.AC.UK/MUSIC/THEBARONYORGAN/THEBARONYORGAN-

TECHNICALSPECIFICATIONS/

[ACCESSED 25 OCT. 2016].

Amichai, E., Blumrosen, G. \& Yovel, Y. (2015) Calling louder and longer: how bats use biosonar under severe acoustic interference from other bats, Proc. Biol. Sci. 282 , p. 20152064.

Au, W.W.L. (1993) The Sonar of Dolphins, New York: Springer-Verlag.

Barber, J.R. \& Kawahara, A.Y. (2013) Hawkmoths produce anti-bat ultrasound, Proc. Biol. Sci. 9 , p. 20130161.

Barnes, W.H. (1952) The Contemporary American Organ, New York: J. Fischer \& Bro.

Bonavia-Hunt, N.A. (1947) The Modern British Organ, London: A. Weekes \& Co.

Campbell, E., Galbraith, W. \& Hayward, G. (2006) A new electrostatic transducer incorporating fluidic amplification, IEEE Ultrasonics Symposium, Vancouver, Canada, 1445-1448. 
Caronti, A., Caliano, G., Iula, A. \& Pappalardo, M. (2002) An accurate model for capacitive micromachined ultrasonic transducers, IEEE Trans. Ultrason. Ferroelectr. Freq. Control, 49 (2), 159-168.

Fletcher, N.H. (1977) Scaling rules for organ flue pipe ranks, Acustica, 27 , pp. 131-138.

Fletcher, N.H. \& Rossing, T.D. (1991) Physics of Musical Instruments, New York: Springer-Verlag.

Hamid, S.B.A. (2013) Enhancing signal to noise ratio for electrostatic transducers, $\mathrm{PhD}$ thesis, University of Strathclyde.

Helmholtz, H.L.F. (1895) On the Sensations of Tone as a Physiological Basis for the Theory of Music, New York: Longmans, Green, \& Co.

Hopkins, E.J. (1855) The Organ, Its History and Construction, London: Robert Cocks \& Co.

Jones, A.T. (1941) End corrections of organ pipes, J. Acoust. Soc. Am., 12, p. 387.

Kinsler, L.E., Frey, A.R., Coppens, A.B. \& J. V. Sanders (2000) Fundamentals of Acoustics, Chichester: John Wiley and Sons.

Ladabaum, I., Jin, X., Soh, H.T., Atalar, A. \& Khuri-Yakub, B.T. (1998) Surface micromachined capacitive ultrasonic transducers, IEEE Trans. Ultrason. Ferroelectr. Freq. Control, 45 (3), 678-690.

Leighton, T.G. (2007) What is ultrasound?, Prog. Biophys. Mol. Biol., 93, 3-83.

Manthey, W., Kroemer, N. \& Mágori, V. (1992) Ultrasonic transducers and transducer arrays for applications in air, Meas. Sci. Technol., 3, 249-261.

Maurello, M.A., Clarke, J.A. \& Ackley, R.S. (2000) Signature characteristics in contact calls of the white-nosed coati, J. Mammal., 81, 415-421.

McVicker, W.R. (1987) An analytical approach to open, cylindrical organ-pipe scaling from a historical perspective with specific reference to the scaling practices of selected organ-builders, $\mathrm{PhD}$ thesis, Durham University.

Nolle, A.W. (1979) Some voicing adjustments of flue organ pipes, J. Acoust. Soc. Am., 66, 1612-1626.

Rucz, P. (2015) Innovative Methods for the Sound Design of Organ Pipes, $\mathrm{PhD}$ thesis, Budapest University of Technology and Economics. 
Schindel, D.W., Hutchins, D.A., Zou, L. \& Sayer, M. (1995) The design and characterization of micromachined air-coupled capacitance transducers, IEEE Trans. Ultrason. Ferroelectr. Freq. Control, 42 (1), 42-50.

Walker, A.J. \& Mulholland, A.J. (2010) A theoretical model of an electrostatic ultrasonic transducer incorporating resonating conduits, IMA J. Appl. Math., 75 (5), 796-810.

Walker, A.J. \& Mulholland, A.J. (2016) A theoretical model of an ultrasonic transducer incorporating spherical resonators, IMA J. Appl. Math., 81 (1), 1-25.

Walker, A.J., Mulholland, A.J., Campbell, E. \& Hayward, G. (2008) A theoretical model of a new electrostatic transducer incorporating fluidic amplification, IEEE Ultrasonics Symposium, Beijing, China, 1409-1412.

Warring, R.H. \& Gibilisco, S. (1985) Fundamentals of Transducers, Pennsylvania: Tab Books.

Wright, M.C.M. (ed.) (2005) Lecture notes on the Mathematics of Acoustics, London: Imperial College Press. 


\section{A Nomenclature}

The tables below provide a full nomenclature of terms used within the article. It is worth noting that, as far as notation is concerned, the available literature is not consistent and care should be taken when comparing with other work.

\begin{tabular}{ll}
\hline Notation & Description \\
\hline$\alpha$ & Attenuation coefficient \\
$\beta$ & Transformation factor \\
$\gamma$ & Cross-sectional area of conduit \\
$\varepsilon_{0}$ & Permitivitty of free space \\
$\varepsilon_{r}$ & Relative permitivitty of mylar membrane \\
$\Xi$ & Displacement of mylar membrane (frequency domain) \\
$\xi$ & Displacement of mylar membrane (time domain) \\
$\rho_{a}$ & Density of air \\
$\rho_{s}$ & Density of membrane \\
$\omega$ & Angular frequency of sound \\
\hline
\end{tabular}




\begin{tabular}{|c|c|}
\hline Notation & Description \\
\hline$C$ & Capacitance \\
\hline$C_{0}$ & Value of capacitance $C$ at $\dot{\xi}=0$ \\
\hline$c$ & Speed of sound \\
\hline$d_{e}$ & Distance between electrodes \\
\hline$d_{m}$ & Thickness of mylar membrane \\
\hline$d_{n}$ & Diameter of the $n^{\text {th }}$ pipe in organ designs \\
\hline$F$ & Force on/from radiating surface \\
\hline$f(t)$ & Applied external force to membrane (time domain) \\
\hline $\bar{f}(\omega)$ & Applied external force to membrane (frequency domain) \\
\hline$G$ & Static conductance \\
\hline$H_{n}$ & Struve function of order $n$ \\
\hline$h$ & Halving number \\
\hline$I$ & Current at electrical inputs \\
\hline$J_{n}$ & Bessel function of the first kind of order $n$ \\
\hline$j$ & Imaginary number \\
\hline$k$ & Wavenumber of sound \\
\hline$L$ & Distance between electrode and membrane \\
\hline$l_{c}$ & Length of cavity \\
\hline$l_{p}$ & Length of conduit \\
\hline$N$ & Number of rows of conduits \\
\hline$n_{j}$ & Number of conduits in a row \\
\hline$P_{o}$ & Pressure produced at membrane load \\
\hline$P(t)$ & Incoming pressure wave (time domain) \\
\hline $\bar{P}(\omega)$ & Incoming pressure wave (frequence domain) \\
\hline RFR & Reception force response \\
\hline$R_{p}$ & Mechanical radiation impedance \\
\hline$R_{v}$ & Damping constant \\
\hline$r_{p}$ & Radius of conduit \\
\hline$S_{c}$ & Surface area of cavity \\
\hline$S_{m}$ & Surface area of membrane \\
\hline$T$ & Transduction Coefficient \\
\hline TVR & Transmission voltage response \\
\hline$V_{a c}$ & Alternating current voltage (time domain) \\
\hline $\bar{V}_{a c}$ & Alternating current voltage (frequency domain) \\
\hline$V_{c}$ & Volume of cavity \\
\hline$V_{d c}$ & Direct current voltage \\
\hline$x_{d c}$ & Direct current deflection of membrane \\
\hline$Z_{E B}$ & Blocked electrical impedance \\
\hline$Z_{m}$ & Mechanical impedance of entire system \\
\hline$Z_{m}^{c}$ & Mechanical impedance of cavity \\
\hline$Z_{m o}^{m}$ & Open-circuit mechanical impedance \\
\hline$Z_{s}^{c}$ & Specific acoustic impedance of cavity \\
\hline$Z_{s}^{l}$ & Specific acoustic impedance of fluid at load \\
\hline$Z_{s}^{m}$ & Specific acoustic impedance of entire system \\
\hline$Z_{s}^{p}$ & Specific acoustic impedance of conduit \\
\hline$Z^{p}$ & Acoustic impedance of cavity \\
\hline
\end{tabular}

